\title{
Expression of ERCC1, RRM1 and LRP in Non-small Cell Lung Cancers and their Influence on Chemotherapeutic Efficacy of Gemcitabine Concomitant with Nedaplatin
}

\author{
Zhen-Qin Qiu' ${ }^{1 *}$ Kun Zhao ${ }^{2}$
}

\begin{abstract}
Objective: To explore the clinical efficacy of gemcitabine concomitant with nedaplatin and drug resistance in the treatment of non-small cell lung cancer (NSCLC) and associated molecular predicators. Materials and Methods: A total of 68 patients diagnosed with NSCLC by histology served as the study objects and were randomly divided into an observation group treated with gemcitabine concomitant with nedaplatin and a control group with cisplatin concomitant with gemcitabine, 34 cases for each group. Short-term and long-term efficacies, adverse responses as well as the expression of nucleotide excision repair cross complementing 1 (ERCC1), ribonucleotide reductase subunit M1 (RRM1) and lung resistance-related protein (LRP) in NSCLC tissues in both groups were assessed. Results: The short-term objective response rate (ORR) and disease control rate (DCR) were 35.3\% (12/34) and 76.5\% (26/34) in the observation group and 38.2\% (13/34) and 85.3\% (29/34) in the control group, respectively, the differences not being statistically significant. The time to progression (TTP) in both groups were 1 12 months, while the median TTP was $135 \mathrm{~d}$ and $144 \mathrm{~d}$, respectively. Though the survival was slightly higher in the control group, there were no significant differences in TTP and survival time. The rates of decreased hemoglobin, vomiting and nausea as well as renal toxicity were evidently lower in the observation group, while other adverse responses demonstrated no significant difference. The positive expression rates of ERCC1, RRM1 and LRP were $47.1 \%(16 / 34), 61.8 \%(21 / 34)$ and $64.7 \%(22 / 34)$ in the observation group, respectively. Compared with negative ERCC1 expression, ORR had decreasing trend and the overall survival time (OS) decreased significantly in patients with positive ERCC1 expression, which were markedly decreased by the positive expressions of RRM1 and LRP. Conclusions: Gemcitabine concomitant with nedaplatin has significant effects in the treatment of NSCLC, with an adverse response rate obviously lower than for cisplatin concomitant with gemcitabine, suggesting that wider use in the clinic is warranted. Additionally, the positive expressions of ERCC1, RRM1 and LRP may increase patient drug resistance, so they can be applied as the chemotherapeutic predicators to guide individualized therapy of NSCLC patients.
\end{abstract}

Keywords: Non-small cell lung cancer - gemcitabine - nedaplatin - drug resistance - ERCC1 - RRM1 - LRP

Asian Pac J Cancer Prev, 15 (17), 7303-7307

\section{Introduction}

Non-small cell lung cancer (NSCLC), as a common malignant tumor accounting for $80 \% \sim 85 \%$ in all lung cancers, is characterized by higher morbidity, dormant onset, rapid development and short survival time, etc., in which $80 \%$ of patients have lost the optimal operative opportunities when diagnosed (Siegel et al., 2012). At present, the primary therapies for NSCLC patients are chemotherapies, in which the platinum-based ones are best in efficacy. However, they are always accompanied with severe gastrointestinal responses as well as renal and hematological toxicities.

Since the development of new-type anti-tumor drugs, the double-drug protocols of the third generation of antitumor chemotherapeutical drugs gemcitabine combined with platinums have become the standard first-line treatments for NSCLC patients with decreased adverse responses to some extent. Though the effective rate is less than $40 \%$, and the drug resistance is still the principal factor that influences the clinical efficacy of chemotherapies (Rossi et al., 2001). Therefore, it is probable that the application of NSCLC drug resistance associated biological predicators to predicate the chemotherapeutic efficacy can apparently improve the therapeutic effective rate and prognosis as well as obviously reduce the unnecessary adverse responses and financial burdens so as to realize the individualized therapies for NSCLC patients. In this study, gemcitabine combined with cisplatin was applied as control group to explore the clinical efficacy and adverse responses of gemcitabine concomitant with nedaplatin and detect the expressions

${ }^{1}$ Unified Supply Center, ${ }^{2}$ Department of Oncology, The 89th Hospital of Chinese People's Liberation Army, Weifang, Shandong, China *For correspondence: qzqsxg qzqsxg@163.com 
of nucleotide excision repair cross complementing 1 (ERCC1), ribonucleotide reductase subunit M1 (RRM1) and lung resistance-related protein (LRP) in NSCLC tissues, aiming to investigate the relationship between ERCC1, RRM1, LRP and chemotherapy associated drug resistances in the hope of seeking a better therapeutic protocol with high efficacy and low toxicity as well as drug resistance associated molecular predicators.

\section{Materials and Methods}

\section{General data}

A total of 68 patients diagnosed with NSCLC by histology from January 2012 to June 2013 served as study objects, in which 47 were males and 21 were females, aged 39 77 years, with median age being 56 years; 14 with initial treatments, 54 with retreatments; 39 with adenocarcinoma, 27 with squamous carcinoma and 2 with large cell carcinoma; clinical stage: 22 in phase IIIB and 46 in phase IV; 15 in higher differentiation, 32 in moderate differentiation and 21 in low differentiation; 23 with lymph node metastasis; all patients had normal blood routine, hepatorenal functions and electrocardiograms; Karnofsky performance status (KPS) score $>70$ scores; expected survival time $>3$ months. The patients were randomly divided into observation group and control group, 34 cases for each group. There were no significant differences between two groups in general data such as age, genders, KPS scores and tumor types $(P>0.05)$.

\section{Therapeutic methods}

Observation group: gemcitabine concomitant with nedaplatin. Administrative protocols: Intravenous injection of $80 \mathrm{mg} / \mathrm{m} 2$ nedaplatin (Qilu Pharmaceutical Co., Ltd, Approval No. 20050563) + 100 mL normal saline (NS) on $\mathrm{d} 1$ for $\geq 1 \mathrm{~h}$, before which $10 \mathrm{mg}$ dexamethasone (Tainjin King York Group HuBei Tianyao Pharmaceutical Co., Ltd, Approval No. H12020514) was routinely given to prevent allergy; Intravenous injection of $1000 \mathrm{mg} / \mathrm{m} 2$ gemcitabine (Jiangsu Hansoh Pharmaceutical Co., Ltd, Approval No. H20030104) $+100 \mathrm{~mL} \mathrm{NS}$ for $\geq 30 \mathrm{~min}$ on d 1,8 and $15,28 \mathrm{~d}$ as a cycle.

Control group: Gemcitabine combined with cisplatin. Administrative protocols: Intravenous injection of $25 \mathrm{mg} /$ m2 ciaplatin (Yunnan Biovalley Pharmaceutical Co., Ltd, Approval No. H20043888) + 100 mL on d 1 3 combining with $2000 \sim 3000 \mathrm{~mL}$ hydrated solution; Gemcitabine was the same with observation group, $28 \mathrm{~d}$ as a cycle.

Before chemotherapy, 5-H3 receptor antagonist (Qilu Pharmaceutical Co., Ltd, Approval No H10970062) was routinely given to prevent vomiting.

\section{Sample collection, disposal and detection}

Sample collection: Samples from operative excision or biopsy were collected, including primary nidi and metastatic lymph node. All samples were fixed by $10 \%$ neutral formaldehyde solution, routinely dehydrated, embedded with paraffin, cut into $4 \mu \mathrm{m}$ slices and routinely stained with heamtoxylin-eosin (HE).

Sample detection: Envision two-step method was applied for Immunohistochemical staining. Antigens were repaired by high temperature and pressure. Endogenous peroxidase was inactivated by $3 \%$ hydrogen peroxide. ERCC1, RRM1 and LRP primary antibodies (rat anti-human ERCC1 monoclonal antibody, rabbit anti-human RRM1 monoclonal antibody, rat anti-human LRP monoclonal antibody, Peking Zhongshan Jinqiao Biotechnology Co., Ltd) were added and cultured in 4 ${ }^{\circ} \mathrm{C}$ refrigerator overnight. Second antibody (PV-9000 detection kit, Peking Zhongshan Jinqiao Biotechnology Co., Ltd) was added and put into $37^{\circ} \mathrm{C}$ dry box for $30 \mathrm{~min}$, then developed with diaminobenzidine (DAB developing kit, Peking Zhongshan Jinqiao Biotechnology Co., Ltd), washed by running water, re-stained with hematoxylin, routinely dehydrated, made into transparent and sealed with neutral gum. The known slices were set as positive control while phosphate buffer solution (PBS) as negative control instead of primary antibody.

\section{Observational indexes}

Short- and long-term clinical efficacies were observed, overall survival time (OS) and tumor time to progression (TTP) were recorded. Adverse response rates and the relationship between the expressions of ERCC1, RRM1 and LRP with clinical efficacy were observed in both groups.

\section{Evaluation criteria}

Evaluation criteria for short-term efficacy by RECIST: (1) Complete response (CR): All target nidus tissue disappeared $>4$ weeks; (2) Partial response (PR): The total of the maximum diameter $\times$ the maximum vertical diameter of nidi decreased $>50 \%$ for $>4$ weeks; (3) Stable disease (SD): The total of the product of the two vertical diameters of nidi decreased $<50 \%$ or increased $<25 \%$ without the development of new nidi for $>4$ weeks; (4) Progressive disease (PD): The total of the product of the two vertical diameters of nidi increased $>25 \%$ or with appearance of new nidi. Objective response rate (ORR) $=\mathrm{CR}+\mathrm{PR}$; Disease control rate $(\mathrm{DCR})=\mathrm{CR}+\mathrm{PR}+\mathrm{SD}$.

Evaluation criteria for positive/negative expressions of ERCC1, RRM1 and LRP: A total of 100 positive cells in tumor cells were calculated under microscopy $(x 400)$. Cells without expressions or positive cell counts $\leq 10 \%$ were determined as negative while positive cell counts $>10 \%$ as positive. Moreover, the sample staining results were evaluated by 2 pathological physicians under doubleblind condition.

\section{Statistical data analysis}

SAS 9.3 statistical software package was used for data analysis. Rates between two groups were compared with $\chi^{2}$ test, while median OS and TTP with Wilcoxon rank sum test. Kaplan-Meier survival curve was adopted for survival analysis and detected with Log-rank test. $P<0.05$ was considered to be statistically significant.

\section{Results}

Comparison of the short-term clinical efficacy

All patients received $\geq 2$ cycles' chemotherapies (totally 234 cycles), with medium cycle amount being 3 
Table 1. Comparisons of Adverse Response Rates Between Two Groups [n(\%)]

\begin{tabular}{lrrrr}
\hline Adverse responses & $\begin{array}{r}\text { Observation } \\
\text { group }\end{array}$ & $\begin{array}{c}\text { Control } \\
\text { group }\end{array}$ & $\chi^{2}$ value & $P$ value \\
\hline Alopecia & $8(23.53)$ & $10(29.41)$ & 0.3022 & 0.5825 \\
Thrombocytopenia & $10(29.41)$ & $12(35.29)$ & 0.2688 & 0.6042 \\
Leucopenia & $18(52.94)$ & $19(55.88)$ & 0.0593 & 0.8076 \\
Decreased hemoglobin & $3(8.82)$ & $14(41.18)$ & 9.4902 & 0.0021 \\
Hepatic toxicity & $1(2.94)$ & $1(2.94)$ & 0.0000 & 1.0000 \\
Renal toxicity & $1(2.94)$ & $6(17.65)$ & 3.9813 & 0.0460 \\
Phlebitis & $4(11.76)$ & $4(11.76)$ & 0.0000 & 1.0000 \\
Myelosuppression & $23(67.65)$ & $29(85.29)$ & 2.9423 & 0.0863 \\
Vomiting and nausea & $9(26.47)$ & $24(70.59)$ & 13.2468 & 0.0003 \\
\hline
\end{tabular}

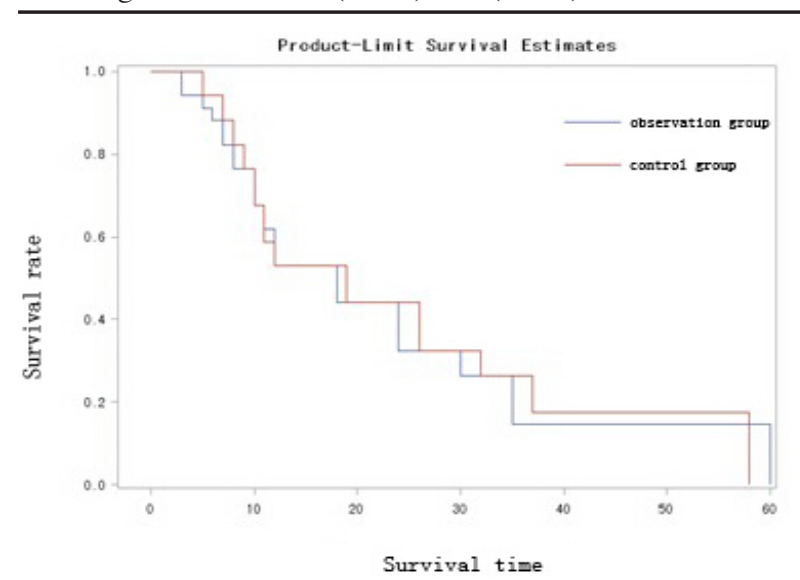

Figure 1. Survival Curves of the Two Groups

(2 6). After treatment, CR, PR, SD, PD, ORR and DCR were $2.94 \%(1 / 34), 32.35 \%(11 / 34), 41.18 \%(14 / 34)$, $23.53 \%(8 / 34), 35.29 \%(12 / 34)$ and $76.47 \%(26 / 34)$ in observation group and were $2.94 \%(1 / 34), 35.29 \%$ $(12 / 34), 47.06 \%(16 / 34), 14.71 \%(5 / 34), 38.24 \%(13 / 34)$ and $85.29 \%(29 / 34)$ in control group, respectively, but there was no significant difference in ORR $\left(\chi^{2}=0.0633\right.$, $P=0.8014)$ and DCR $\left(\chi^{2}=0.8559, P=0.3549\right)$.

Comparison of the long-term clinical efficacyb

The TTP was 1 12 months in both groups, while the median one was $135 \mathrm{~d}$ and $144 \mathrm{~d}$ in observation group and control group, respectively, and there was no significant difference $(P=0.9414)$. Though the survival curve was slight higher in control group than in observation group, the difference was not significant (Wilcoxon, $P=0.8015$; Log-rank, $P=0.7682$ ) (Figure 1).

\section{Comparisons of adverse response rates}

The main adverse responses induced by chemotherapies were alopecia, thrombocytopenia, leucopenia, reduced hemoglobin, hepatic toxicity, renal toxicity and phlebitis, and the rate comparisons showed that the rates of reduced hemoglobin, vomiting and nausea and renal toxicity were evidently lower in observation group than in control group, but there were no significant differences in other adverse responses such as leucopenia and hepatic toxicity, etc. (Table 1).

Expression of ERCC1, RRM1 and LRP in NSCLC tissues Immunohistochemical positive staining showed brown granules, in which ERCC1, RRM1 and LRP were located in nucleus, cytoplasm and membrane and/or cytoplasm of

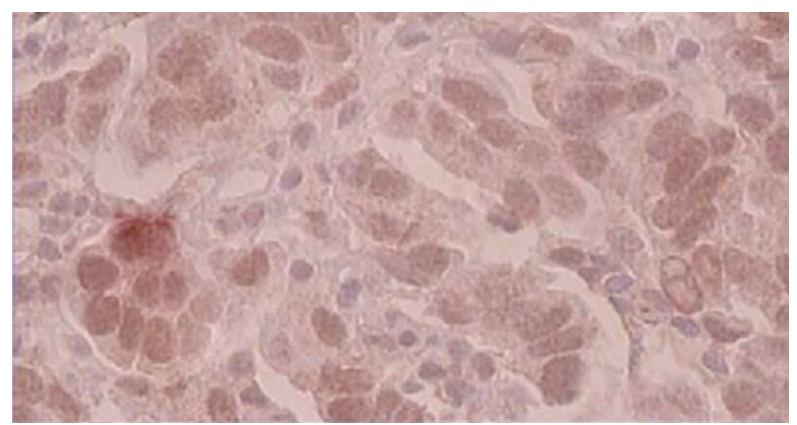

Figure 2. Expression of ERCC1 in NSCLC (SP×400)

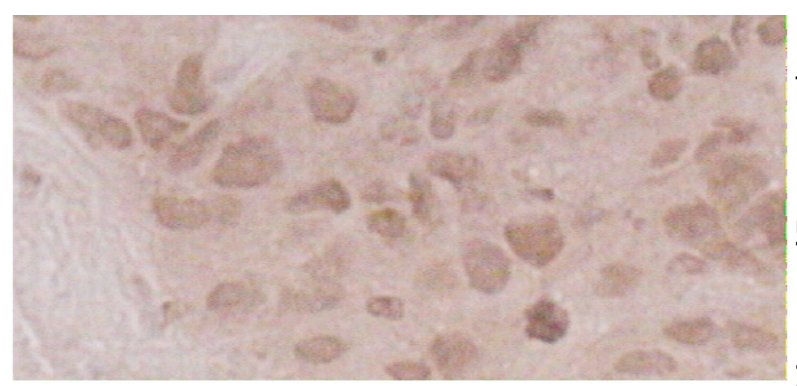

Figure 3. Expression of RRM1 in NSCLC (SP×400)

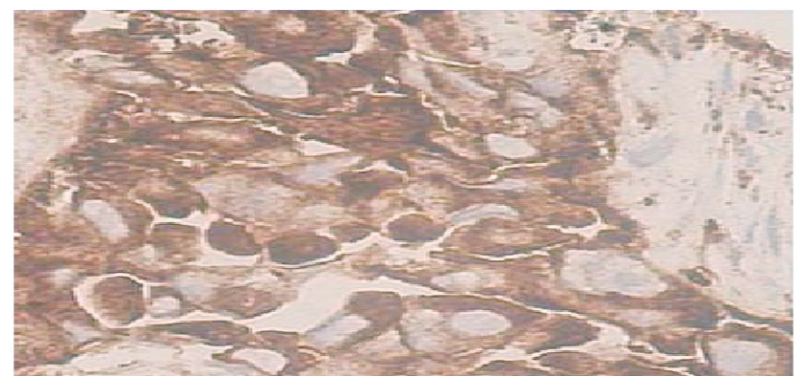

Figure 4. Expression of LRP in NSCLC (SP×400)

Table 2. Relationship Between the Expressions of ERCC1, RRM1, LRP and Short- and Long-term Clinical Efficacies of Gemcitabine Concomitant with Nedaplatin

\begin{tabular}{|c|c|c|c|c|c|c|c|}
\hline & & ORR/n $(\%)$ & $\chi^{2}$ value & $P$ value & $\mathrm{OS} / \mathrm{mon}$ & h $\chi^{2}$ value & $P$ value \\
\hline \multirow[t]{2}{*}{ ERCC1 } & + & $3(18.75)$ & 3.6222 & 0.0570 & 10.5 & 6.4968 & 0.0108 \\
\hline & - & $9(50.00)$ & & & 27 & & \\
\hline \multirow[t]{2}{*}{ RRM1 } & + & $4(19.05)$ & 6.3479 & 0.0118 & 11 & 4.4163 & 0.0356 \\
\hline & - & $8(61.54)$ & & & 24 & & \\
\hline \multirow[t]{2}{*}{ LRP } & + & $5(22.73)$ & 4.3105 & 0.0379 & 11.5 & 5.2071 & 0.0225 \\
\hline & - & $7(58.33)$ & & & 29.5 & & \\
\hline
\end{tabular}

cancer cells, respectively (Figures 2 4). In addition, the positive expression rates of ERCC1, RRM1 and LRP were $47.06 \%$ (16/34), 61.76\% (21/34) and 64.71\% (22/34), respectively.

Relationship between expression of ERCC1, RRM1, LRP and the short-and long-term clinical efficacy

Compared with negative ERCC1 expression, ORR had decreasing trend and overall survival (OS) decreased significantly in patients with positive ERCC1 expression, which were markedly reduced by the positive expressions of RRM1 and LRP, suggesting that the positive expressions of ERCC1, RRM1 and LRP might increase patients' drug resistances to gemcitabine concomitant with nedaplatin (Table 2). 


\section{Discussion}

Lung cancers rank the top of cancer-related death with increasing morbidity around the world (Aydiner et al., 2013; Cai et al., 2013; Kaya et al., 2013; Mutlu et al., 2013; Natukula et al., 2013; Oven et al., 2013; Unal et al., 2013; Wang et al., 2013). At present, the chemotherapeutic protocols for NSCLC mainly apply platinums as basic drugs combining with the third generation chemotherapeutic drugs such as paclitaxel, gemcitabine and vinorelbine, etc.. Multiple randomized clinical trials showed that the first-line treatment of double-drug protocol containing platinum had similar clinical effect on malignant tumors, for which the main differences were toxic characteristics and treatment costs (Sugiyama et al., 2011; Teramoto et al., 2012; Wang et al., 2012; He et al., 2013). Gemcitabine, a new-type of deoxycytidine analogue, can not only be used to inhibit ribonucleotide reductase to form hidden chains but also to improve cell apoptosis by rupturing the synthesis of DNA double chains. It was reported that the medium OS of single gemcitabine was 8 months (Valle et al., 2010; Ueno et al., 2013), but the effective response and OS could both be markedly improved by gemcitabine combined with platinums. Nedaplatin is also called cis-Dichlorobis (ethylenediamine) platinum (II). After entering into cell, the chains between alcohol oxygen on lipid radicals of glycolic acid and platinums are ruptured, which leads to the combination of water and platinum, then the ionic substances are formed. The ruptured radical lipids are unsteadily released and form multiple ionic substances that can combine with DNA to inhibit DNA duplication, thus achieving the anti-tumor effect. A study found that nedaplatin concomitant with gemcitabine had no significant difference in clinical efficacy when compared with the combination with ciaplatin, but had decreased rates of gastrointestinal responses and renal toxicity, and was still effective on patients with resistance to cisplatin (Sugiyama et al., 2011).

In this study, gemcitabine combined with cisplatin were applied as control group to explore the clinical efficacy and adverse responses of gemcitabine concomitant with nedaplatin, whose results demonstrated that the short- and long-term clinical efficacies had no significant differences, but the rates of decreased hemoglobin, vomiting and nausea and renal toxicity were evidently lower in observation group than in control group, being consistent with the results of another study (Sugiyama et al., 2011).

Drug resistance is one of the primary reasons for chemotherapeutic failure, and there is no effective method to guide the selection of chemotherapeutic protocols. The abnormal synthesis and repair of DNA are closely related with the development and multi-drug resistance of tumors, in which nucleotide repair system (NER) is the primary mechanism for repairing the drug-induced DNA damages. ERCC1, as an important member of ENR families and rate-limiting enzyme of ENR, involves in the recognition of DNA damage and the incision of DNA chains. However, its over-expression can rapidly repair the damaged DNA in $\mathrm{G} 2 / \mathrm{M}$ phase, bringing about resistances to gemcitabine and cisplatin (Wang et al., 2010; Jiang et al., 2012). RRM1, as a tumor suppressor gene and the molecular target of gemcitabine, can control the specificity of substrate. In vitro experiment proved that RRM1 expression was in association with the sensitivity of gemcitabine (Bepler et al., 2006). Meanwhile, another study also confirmed that in vivo, RRM1 expression was in close relationship with the clinical efficacy of chemotherapies based on gemcitabine (Lee et al., 2010). LRP, as an important resistance protein in muiti-drug resistances, can transfer the drugs in nucleus into vesicles in cytoplasm or directly outside of cells so as to avoid the nucleus DNA damages and the development of drug resistance. Moreover, it was proved that LRP expressed in multiple tumors and was closely connected with the sensitivities of tumors to the chemotherapeutic drugs (Dalla-Torre et al., 2007). Nonetheless, the detailed report on the relationship between the expressions of ERCC1, RRM1 as well as LRP and the chemotherapeutic efficacy of gemcitabine concomitant with nedaplatin is still rare.

This study also detected the expressions of ERCC1, RRM1 and LRP in NSCLC tissues, and primarily explored their relationship with gemcitabine concomitant with nedaplatin. The results of this study indicated that the positive expressions of ERCC1, RRM1 and LRP could reduce the short- and long-term clinical efficacies of gemcitabine concomitant with nedaplatin, which was predicated to be correlated with the increase of patients' drug resistance, suggesting that the expressions of ERCC1, RRM1 and LRP could be considered as the drug resistance associated molecular predicators and chemotherapeutic predicators, so they can be applied as chemotherapy predicators to guide the individualized therapy of NSCLC.

To sum up, this study revealed that gemcitabine concomitant with nedaplatin had similar short- and long-term clinical efficacies to gemcitabine combined with cisplatin, but were more suitable for patients with advanced ages and poor constitution due to the slight adverse responses. And the detection of the expressions of ERCC1, RRM1 and LRP could predicate the prognosis and guide clinical application to some extent. However, because of the limited study samples in this study, more large-scale and multi-center clinical studies are needed to verify the clinical efficacy, adverse responses and molecular predicators of gemcitabine concomitant with nedaplatin.

\section{References}

Aydiner A, Ciftci R, Karabulut S, et al (2013). Does beta-blocker therapy improve the survival of patients with metastatic non-small cell lung cancer? Asian Pac J Cancer Prev, 14, 6109-14.

Bepler G, Kusmartseva I, Sharma S, et al (2006). RRM1 modulated in vitro and in vivo efficacy of gemcitabine and platinum in non-small-cell lung cancer. J Clin Oncol, 24, 4731-7.

Cai Y, Wang JY, Liu H (2013). Clinical observation of whole brain radiotherapy concomitant with targeted therapy for brain metastasis in non-small cell lung cancer patients with chemotherapy failure. Asian Pac J Cancer Prev, 14, 5699-703.

Dalla-Torre CA, de Toledo SRC, Yoshimoto M, et al (2007). 
Expression of major vault protein gene in osteosarcoma patients. J Orthop Res, 25, 958-63.

He YF, Ji CS, Hu B, et al (2013). A phase II study of paclitaxel and nedaplatin as front-line chemotherapy in Chinese patients with metastatic esophageal squamous cell carcinoma. World J Gastroenterol, 19, 5910.

Jiang J, Liang X, Zhou X, et al (2012). ERCC1 expression as a prognostic and predictive factor in patients with non-small cell lung cancer: a meta-analysis. Mol Biol Rep, 39, 6933-42.

Kaya V, Yildirim M, Demirpence O, et al (2013). Prognostic significance of basic laboratory methods in non- small-celllung cancer. Asian Pac J Cancer Prev, 14, 5473-6.

Lee JJ, Maeng CH, Baek SK, et al (2010). The immunohistochemical overexpression of ribonucleotide reductase regulatory subunit M1 (RRM1) protein is a predictor of shorter survival to gemcitabine-based chemotherapy in advanced non-small cell lung cancer (NSCLC). Lung Cancer, 70, 205-10.

Mutlu H, Buyukcelik A, Aksahin A, et al (2013). Does sunlight exposure improve survival in patients with non-small cell lung cancer? Asian Pac J Cancer Prev, 14, 6301-4.

Natukula K, Jamil K, Pingali UR, et al (2013). The codon 399 $\mathrm{Arg} / \mathrm{Gln} \mathrm{XRCC} 1$ polymorphism is associated with lung cancer in Indians. Asian Pac J Cancer Prev, 14, 5275-9.

Oven Ustaalioglu BB, Unal OU, Turan N, et al (2013). Prognostic factors for lymph node negative stage I and IIA non-small cell lung cancer: multicenter experiences. Asian Pac J Cancer Prev, 14, 6287-92.

Rossi D, Graziano F, Catalano V, et al (2001). A new cisplatin/ gemcitabine schedule in locally advanced (IIIB) and metastatic (IV) non-small cell lung cancer: relationship between dose-intensity and efficacy. A phase II study. Anticancer Res, 22, 3087-92.

Siegel R, Naishadham D, Jemal A (2012). Cancer statistics, 2012. CA Cancer J Clin, 62, 10-29.

Sugiyama T, Hirose T, Nakashima M, et al (2011). Evaluation of the efficacy and safety of the combination of gemcitabine and nedaplatin for elderly patients with advanced non-small-cell lung cancer. Oncology, 81, 273-80.

Teramoto K, Asada Y, Ozaki Y, et al (2012). A phase II study of docetaxel plus nedaplatin in patients with metastatic non-small-cell lung cancer. Cancer Chemother Pharmacol, 70, 531-7.

Ueno H, Ioka T, Ikeda M, et al (2013). Randomized phase III study of gemcitabine plus S-1, S-1 alone, or gemcitabine alone in patients with locally advanced and metastatic pancreatic cancer in Japan and Taiwan: GEST study. J Clin Oncol, 31, 1640-8.

Unal D, Eroglu C, Kurtul N, et al (2013). Are neutrophil/ lymphocyte and platelet/lymphocyte rates in patients with non-small cell lung cancer associated with treatment response and prognosis? Asian Pac J Cancer Prev, 14, 5237-42.

Valle J, Wasan H, Palmer D H, et al (2010). Cisplatin plus gemcitabine versus gemcitabine for biliary tract cancer. $N$ Engl J Med, 362, 1273-81.

Wang X, Zhao J, Yang L, et al (2010). Positive expression of ERCC1 predicts a poorer platinum-based treatment outcome in Chinese patients with advanced non-small-cell lung cancer. Med Oncol, 27, 484-90.

Wang JY, Cai Y (2013). Clinical observation and prognostic analysis of pemetrexed plus platinum as first-line treatment in patients with advanced non-small cell lung cancer. Asian Pac J Cancer Prev, 14, 6267-71.

Wang M, Gu J, Wang HX, et al (2012). Retrospective study of gemcitabine based chemotherapy for unresectable or recurrent esophagus squamous cell carcinoma refractory to first line chemotherapy. Asian Pac J Cancer Prev, 13, 4153-6. 